\title{
Multivariate analysis of morphological characteristics of two species of passion flower with ornamental potential and of hybrids between them
}

\author{
E.A. Santos ${ }^{1}$, M.M. Souza ${ }^{1}$, A.P. Viana ${ }^{2}$, A.A.F. Almeida ${ }^{1}$, J.C.O. Freitas ${ }^{1}$ \\ and P.R. Lawinscky ${ }^{1}$ \\ ${ }^{1}$ Departamento de Ciências Biológicas, Universidade Estadual de Santa Cruz, \\ Ilhéus, BA, Brasil \\ ${ }^{2}$ Centro de Ciências e Tecnologia Agropecuária, \\ Universidade Estadual do Norte Fluminense Darcy Ribeiro, \\ Campos dos Goytacazes, RJ, Brasil \\ Corresponding author: M.M. Souza \\ E-mail: souzamagg@yahoo.com.br
}

Genet. Mol. Res. 10 (4): 2457-2471 (2011)

Received December 14, 2010

Accepted May 5, 2011

Published October 13, 2011

DOI http://dx.doi.org/10.4238/2011.October.13.3

\begin{abstract}
We estimated genetic parameters through multivariate analysis of two species of Passiflora and their hybrids, considered of ornamental potential, based on the morphological characteristics: flower diameter, corona diameter, corona filament size, flower peduncle length, petal length and width, sepal length and width, internode length, stem diameter, leaf length, leaf width $(\mathrm{mm})$, and leaf area $\left(\mathrm{cm}^{2}\right)$. Five specimens of Passiflora sublanceolata [ex P. palmeri var. sublanceolata], five of $P$. foetida var. foetida and $20 \mathrm{~F}_{1}$ hybrids between the two were evaluated. A randomized block design with four replications was used. The data were submitted to variance analysis and multivariate procedures, principal components analysis and unweighted pair group method with arithmetic mean grouping. We found significant differences between genotypes for all these morphological parameters.
\end{abstract}


The hybrid plants had the highest variability, making them the most indicated for future improvement programs. The various multivariate techniques gave similar results, allowing separation of the plants into three distinct groups, these being the two paternal species and the hybrids. The hybrids were closer to the male genitors, revealing a paternal effect on the inheritance of vegetative and floral characters. Based on estimates of genetic parameters, the floral characteristics are the most indicated for the selection of plants for ornamental purposes, since these characteristics displayed greatest variability, a variation index of more than one, and high genotypic determination coefficients.

Key words: Crop breeding; Interspecific hybrids; Passifloraceae; Ornamental Passiflora

\section{INTRODUCTION}

The genus Passiflora is represented by more than 500 species (Cervi, 2005); however, new species continue to be found (Cervi, 2006; Nunes and Queiroz, 2007; Viana, 2009). There is a high incidence of this genus in the Brazilian flora, with approximately 150 native species (Faleiro et al., 2005), showing a large available potential as germplasm source for genetic improvement. The efficient management of this germplasm is extremely important for the scientist who needs a well-characterized germplasm for use in research and breeding.

In order to develop a genetic improvement program, the first step is to characterize the germplasm to obtain basic information about the important characteristics of the species, lines, variety, or hybrid. The success of an improvement program depends mainly on the choice of the germplasm. A well-made choice provides populations with the potential to supply superior progeny, for more efficiency of the breeding program. With regard to Passiflora species and improvement with ornamental potential, the choice is directed at the varieties that display interesting characteristics for the current ornamental plant market. Thus, many species of Passiflora show flowers with exotic shapes, strong or soft colors, and exuberant foliage, traits that enable their inclusion in the ornamental plant agribusiness (Abreu et al., 2009). As these plants are essentially allogamous it is possible to use various genetic improvement methods, either by means of mass selection, aiming to increase the frequency of favorable genes, or by means of interspecific hybridization to explore the hybrid vigor (Bruckner, 1997).

Characteristics of interest are improved by means of interspecific hybridizations in other countries where passion fruit is essentially ornamental. By this process, many hybrids that are adequate for ornamental use have already been obtained (Ulmer and MacDougal, 2004), either as landscaping solution for large areas such as parks and gardens, or as potted plants for decoration of porches and interiors (Peixoto, 2005).

Passiflora improvement programs developed in Brazil are basically directed at yellow passion fruit, aiming to meet market demands as regards productivity, fruit quality and resistance to diseases, as the improvement of this culture is directly related to fruit currently more important in the domestic market in some countries (Meletti et al., 2005). Therefore, programs aimed at the use of this species as ornamental plants have been neglected. In the USA and European countries, Passiflora are largely used for the decoration of gardens and greenhouses 
and for interior decorating (Rushing, 2003; Abreu et al., 2009).

In this sense, biometric analysis, such as estimation of genetic parameters, has been essential for breeding programs. Information on genotypic variance, heritability and variation rate allow the identification and quantification of the nature of the action of genes involved in the control of a certain trait, so as to evaluate the efficiency of different improvement strategies (Cruz and Carneiro, 2003). Although the estimation of genetic parameters provides such information, extremely useful for plant breeders, studies of this kind for the genetic improvement of passifloras as ornamental plants have not been developed, whereas there are many researchs on the cultivated species (Viana et al., 2004; Moraes et al., 2005; Gonçalves et al., 2007; Silva et al., 2009). In passion fruit plants, multivariate analysis is applied especially in genetic divergence studies (Crochemore et al., 2003; Primot et al., 2005; Cerqueira-Silva et al., 2009; Viana et al., 2010).

This study aimed to characterize genitors and $\mathrm{F}_{1}$ hybrids of Passiflora with ornamental potential, to evaluate the genetic divergence between genotypes and to estimate genetic parameters by using 14 descriptors, for determining their potential for genetic improvement of ornamental plants for interior decorating.

\section{MATERIAL AND METHODS}

\section{Plant material and cultivation conditions}

Hybrid plants of the $\mathrm{F}_{1}$ progeny obtained from the cross of the genitors Passiflora sublanceolata (Killip) J.M. MacDougal (ex P. palmeri var. sublanceolata Killip) and P. foetida var. foetida $\mathrm{L}$. were used. The $\mathrm{F}_{1}$ hybrid progeny called HD13 comprised 20 plants (HD13 101, HD13 102, HD13 103, HD13 105, HD13 107, HD13 115, HD13 123, HD13 124, HD13 125, HD13 133, HD13 134, HD13 135, HD13 136, HD13 137, HD13 139, HD13 140, HD13 141, HD13 145, HD13 146, and HD13 147), which were evaluated together with five plants of each genitor species: P. foetida var. foetida, donated by Universidade Estadual do Norte Fluminense Darcy Ribeiro, Campos dos Goytacazes, RJ, and P. sublanceolata from EMBRAPA Cerrados, Brazil. Genitors were kept at the Active Germplasm Bank located at coordinates $14^{\circ}$ $39^{\prime} \mathrm{S}, 39^{\circ} 10^{\prime} \mathrm{W}$; $78 \mathrm{~m}$ a.s.l.

Five genotypes of each genitor plant and from each one of the 20 hybrid genotypes were asexually propagated. The cuttings were taken from the intermediate part of the branch, prepared and standardized with four nodes and four leaves reduced to half their area. After bevel sectioning, the basal extremities were immersed in talc powder (inert powder) containing synthetic auxin (indole-3 butyric acid - IBA) at a concentration of $2 \mathrm{~g} / \mathrm{kg}$. The cuttings were put in black 1.5-L polyethylene bags containing washed sand. During the rooting period, the cuttings were kept in a greenhouse and irrigated twice a day. After the rooting, the cuttings were transferred to 42-L plastic pots filled with a sandy-argillaceous A-horizon soil, and transferred to an experimental area.

The experiment was established in February 2007 under field conditions. The experiment consisted of a randomized block design, with 30 genotypes in four replications. The plants were managed in vertical cordon with two wire threads starting $1.50 \mathrm{~m}$ from the soil, with spaces of $0.5 \mathrm{~m}$ between rows and $1.0 \mathrm{~m}$ between plants. The crop management used followed the technical recommendations for passion fruit (Manica, 1981). 


\section{Interspecific hybridization}

The interspecific crosses were carried out in January 2006 always from 7:30 am to 9:00 am, the time during which flowers open. Five plants of $P$. foetida var. foetida were used as male genitor and five $P$. sublanceolata as female genitor. Genitor flower buds in pre-anthesis were protected with paper bags one day prior to hybridization. The buds belonging to the female genitors were emasculated. On the following morning, the anthers of $P$. foetida var. foetida were collected in bulk and the pollen grains were deposited on the stigmas of $P$. sublanceolata, by carefully rubbing the anther on the stigma using tweezers. After artificial hybridization, the crosses were identified and the flowers were again protected for up to $24 \mathrm{~h}$. The fruits were protected with nylon mesh until complete ripening. The seeds were dried at room temperature, put into paper bags encapsulated by plastic bags and kept at $\pm 10^{\circ} \mathrm{C}$. All seeds were sown in Styrofoam trays for germinating with 128 holes, containing sand and cow manure in a proportion of 1:1 as substrate and kept in greenhouses. Twenty hybrid plants were obtained.

\section{Morphological characteristics}

The morphological evaluations were carried out from July to November of 2007. Due to the lack of ornamental morphological descriptors for Passiflora at the time of the research, the following characteristics were analyzed: i) internode length (IL), based on the extremities of the main axis nodes; ii) stem diameter (SD), above the nodes of the main axis; iii) leaf length (LL), longitudinal measurement of larger extremity; iv) leaf width (LW), size of largest dimension; v) leaf area (LA); vi) flower diameter (FD), based on the extremities of the flower; vii) corona diameter $(\mathrm{CD})$, based on the extremities of the corona filaments; viii) flower peduncle length (FPL), from the insertion in the flower's receptacle to the insertion on the stem; ix) first (SF1) and $\mathrm{x}$ ) second (SF2) size of outer series of corona filaments, from the insertion in the flower's receptacle to the apex; $\mathrm{xi}$ ) petal length (PL), from the insertion in the flower to the apex; xii) petal width (PW), size of the largest dimension; xiii) sepal length (SL), from the insertion in the flower to the apex, and xiv) sepal width (SW), size of the largest dimension. The measurements were made with a digital caliper and leaf area meter LI-3100 (Li-Cor, USA).

\section{Statistical analyses}

The computer program Genes (Cruz, 2006) was used to carry out variance analysis, genetic parameter estimates, Scott-Knott means grouping test $(\mathrm{P}<0.05)$, principal component analysis, and estimation of distance matrices; program R - "Project for Statistical Computing" (open source), Mega 4, was used for constructing diagrams with the ascending hierarchical classification algorithm UPGMA (unweighted pair grouped method average) and for preparing graphs.

The genetic parameters were estimated based on the variance analysis scheme and expected mean squares according to the following statistical model:

$$
\mathrm{Y}_{\mathrm{ijk}}=\mu+\mathrm{B}_{\mathrm{j}}+\mathrm{G}_{\mathrm{i}}+\varepsilon_{\mathrm{ijk}}
$$

where: $\mu=$ general mean; $G_{i}=$ effect of the $\mathrm{i}$-th genotype as fixed $(\mathrm{i}=1,2, \ldots \ldots . . \mathrm{g}) ; B_{j}=$ effect 
of the k-th block $(\mathrm{k}=1,2, \ldots \ldots, \mathrm{r}) ; \varepsilon_{i j k}=$ experimental error, related to each observation, assumption NID $\left(0, \sigma^{2}\right)$.

\section{Estimates of genetic parameters}

The expected mean squares estimates were obtained from the variance analysis of each characteristic. The estimated parameters were:

a) Phenotypic variance $\left(\sigma_{f}^{2}\right)$

$$
\sigma_{\mathrm{f}}^{2}=\frac{G M S}{r}
$$

b) Genotypic variability $\left(\Phi_{g}\right)$

$$
\Phi_{g}=\frac{G M S-R M S}{r}
$$

c) Residual variance $\left(\sigma_{e}^{2}\right)$

$$
\sigma_{\mathrm{e}}^{2}=\frac{R M S}{r}
$$

d) Genotypic determination coefficient $\left(H^{2}\right)$

$$
H^{2}=\frac{G M S-R M S}{G M S}
$$

e) Experimental variation coefficient $\left(V C_{e}\right)$

$$
V C_{e}=\frac{100 \times \sqrt{\sigma_{e}}}{m}
$$

f) Genotypic variation coefficient $\left(V C_{g}\right)$

$$
V C_{g}=\frac{100 \times \sqrt{\sigma_{g}}}{m}
$$




\section{g) Variation index $(V I)$}

$$
V I=\frac{V C_{g}}{V C_{e}}
$$

\section{RESULTS}

The summaries of variance analyses with regard to mean squares, averages and experimental variation coefficients of vegetative and floral morphological characteristics are presented in Tables 1 and 2, respectively. By means of the F-test $(\mathrm{P}<0.01)$, a significant difference was found between genotypes for all descriptors evaluated, indicating the existence of genetic variability among genotypes, and a favorable situation towards improvement for obtaining superior genotypes for the characteristics under study.

Table 1. Mean squares, averages and experimental variation coefficients $\left(V C_{e}\right)$ for five vegetative morphological
characteristics for genitors Passiflora sublanceolata and $P$. foetida var. foetida and $\mathrm{HD} 13 \mathrm{~F}_{1}$ hybrids.
\begin{tabular}{llllccc}
\hline SV & d.f. & \multicolumn{5}{c}{ Mean squares } \\
\cline { 2 - 6 } & & $\mathrm{IL}(\mathrm{mm})$ & $\mathrm{SD}(\mathrm{mm})$ & $\mathrm{LL}(\mathrm{mm})$ & $\mathrm{LW}(\mathrm{mm})$ & $\mathrm{LA}\left(\mathrm{cm}^{2}\right)$ \\
\hline Block & 3 & & & & \\
Genotype & 29 & $142.1770^{* *}$ & $2.5379^{* *}$ & $219.8634^{* *}$ & $112.8869^{* *}$ & $105.7138^{* *}$ \\
Error & 27 & 32.2643 & 0.1075 & 37.1391 & 25.8593 & 24.7107 \\
Mean & & 61.90 & 3.59 & 94.82 & 75.60 & 42.18 \\
$V C_{e}(\%)$ & 9.17 & 9.12 & 6.42 & 6.72 & 11.78 \\
\hline
\end{tabular}

$\mathrm{SV}=$ source of variation; d.f. = degrees of freedom; $\mathrm{IL}=$ internode length; $\mathrm{SD}=$ stem diameter; $\mathrm{LL}=$ leaf length; $\mathrm{LW}=$ leaf width; $\mathrm{LA}=$ leaf area. ${ }^{* *}$ Significant at $1 \%$ probability $(\mathrm{P}<0.01)$ by the $\mathrm{F}$-test.

Table 2. Mean squares, averages and experimental variation coefficients $\left(V C_{e}\right)$ for nine flower morphological characteristics for genitors Passiflora sublanceolata and $P$. foetida var. foetida, and HD13 $\mathrm{F}_{1}$ hybrids.

\begin{tabular}{|c|c|c|c|c|c|c|c|c|c|c|}
\hline \multirow[t]{2}{*}{ SV } & \multirow[t]{2}{*}{ d.f. } & \multicolumn{9}{|c|}{ Mean squares } \\
\hline & & $\mathrm{FD}(\mathrm{mm})$ & $\mathrm{CD}(\mathrm{mm})$ & $\mathrm{SF} 1(\mathrm{~mm})$ & $\mathrm{SF} 2(\mathrm{~mm})$ & FPL (mm) & PL (mm) & $\mathrm{PW}(\mathrm{mm})$ & $\mathrm{SL}(\mathrm{mm})$ & $\mathrm{SW}(\mathrm{mm})$ \\
\hline Block & 3 & & & & & & & & & \\
\hline Genotype & 29 & $1248.0181 * *$ & $260.7716^{* * *}$ & $16.0114 * *$ & $8.8492 * *$ & $1053.3904 * *$ & $253.1045^{* *}$ & $9.0327 * *$ & $267.9581 * *$ & $10.7674 * *$ \\
\hline Error & 27 & 1.9753 & 0.2769 & 0.1272 & 0.1859 & 24.6864 & 0.5464 & 0.1258 & 0.7892 & 0.1100 \\
\hline Mean & & 62.77 & 24.71 & 9.52 & 11.23 & 50.07 & 28.31 & 8.75 & 28.28 & 9.06 \\
\hline$V C_{e}(\%)$ & & 2.23 & 2.12 & 3.74 & 3.83 & 9.92 & 2.61 & 4.05 & 3.14 & 3.65 \\
\hline
\end{tabular}

$\mathrm{SV}=$ source of variation; d.f. $=$ degrees of freedom; $\mathrm{FD}=$ flower diameter; $\mathrm{CD}=$ corona diameter; SF1 = size of first outer series of corona filaments; SF2 = size of second outer series of corona filaments; FPL = flower peduncle length; $\mathrm{PL}=$ petal length; $\mathrm{PW}=$ petal width; $\mathrm{SL}=$ sepal length; $\mathrm{SW}=$ sepal width. $* *$ Significant at $1 \%(\mathrm{P}<0.01)$ probability by the F-test.

\section{Evaluation of vegetative and floral morphological characteristics}

The mean values for each genotype related to the evaluated floral and vegetative morphological characteristics were grouped by means of the Scott-Knott test $(\mathrm{P}<0.05)$ (Tables 
3 and 4). With regard to the vegetative characteristics, IL and LW displayed four distribution groups, SD seven, LL five and LA six (Table 3). As to floral characteristics, FD, FPL and SW showed eight groups, CD and SF1 12, SF2 nine, FPL and PL six, and SL seven (Table 4). The results obtained show reduced morphological variability of parents in all characteristics under study; the variability was even lower for the male genitor P. foetida var. foetida, whereas hybrids showed higher variability.

Hybrids had mean values similar to or higher than those of the genitors for the vegetative characteristics IL, SD, LL, LW, and LA (52.82-87.81, 3.06-6.64, 92.89-128.92, 73.3090.66, and 39.93-69.76, respectively) (Table 3). The lowest values for the characteristics IL, LL, LW, and LA were observed for P. sublanceolata genitors (52.02-61.50, 79.76-94.28, 61.96-70.76, and 31.11-39.87, respectively). However, for SD, P. foetida var. foetida genitors showed the lowest means (2.59-2.03) (Table 3).

\begin{tabular}{|c|c|c|c|c|c|}
\hline \multirow[t]{2}{*}{ Genotype } & \multicolumn{5}{|c|}{ Characteristics } \\
\hline & $\mathrm{IL}(\mathrm{mm})$ & $\mathrm{SD}(\mathrm{mm})$ & $\mathrm{LL}(\mathrm{mm})$ & $\mathrm{LW}(\mathrm{mm})$ & $\mathrm{LA}\left(\mathrm{cm}^{2}\right)$ \\
\hline P. sublanceolata 1 & $52.02 \mathrm{~d}$ & $3.87 \mathrm{e}$ & $85.85 \mathrm{e}$ & $67.71 \mathrm{c}$ & $34.52 \mathrm{f}$ \\
\hline P. sublanceolata 2 & $53.48 \mathrm{~d}$ & $3.94 \mathrm{e}$ & $79.76 \mathrm{e}$ & $62.43 \mathrm{~d}$ & $31.11 \mathrm{f}$ \\
\hline P. sublanceolata 3 & $54.08 \mathrm{~d}$ & $3.71 \mathrm{e}$ & $82.53 \mathrm{e}$ & $61.96 \mathrm{~d}$ & $31.43 \mathrm{f}$ \\
\hline P. sublanceolata 4 & $52.35 \mathrm{~d}$ & $3.93 \mathrm{e}$ & $91.14 \mathrm{~d}$ & $70.76 \mathrm{c}$ & $38.92 \mathrm{e}$ \\
\hline P. sublanceolata 5 & $61.50 \mathrm{c}$ & $3.59 \mathrm{e}$ & $94.28 \mathrm{~d}$ & $70.67 \mathrm{c}$ & $39.87 \mathrm{e}$ \\
\hline P. foetida 6 & $62.20 \mathrm{c}$ & $2.29 \mathrm{~g}$ & $89.23 \mathrm{~d}$ & $76.14 b$ & $40.42 \mathrm{e}$ \\
\hline P. foetida 7 & $57.14 \mathrm{~d}$ & $2.37 \mathrm{~g}$ & $91.04 \mathrm{~d}$ & $78.41 \mathrm{~b}$ & $40.88 \mathrm{e}$ \\
\hline P. foetida 8 & $59.58 \mathrm{c}$ & $2.03 \mathrm{~g}$ & $91.42 \mathrm{~d}$ & $81.01 \mathrm{~b}$ & $42.97 \mathrm{e}$ \\
\hline P. foetida 9 & $59.68 \mathrm{c}$ & $2.16 \mathrm{~g}$ & $94.94 \mathrm{~d}$ & $83.26 \mathrm{a}$ & $45.07 \mathrm{~d}$ \\
\hline P. foetida 10 & $61.61 \mathrm{c}$ & $2.59 \mathrm{f}$ & $86.20 \mathrm{e}$ & $76.24 \mathrm{~b}$ & $38.10 \mathrm{e}$ \\
\hline HD13 101 & $75.87 \mathrm{~b}$ & $5.67 \mathrm{~b}$ & $108.52 \mathrm{c}$ & $81.11 \mathrm{~b}$ & $49.67 \mathrm{~d}$ \\
\hline HD13 102 & $73.86 \mathrm{~b}$ & $5.06 \mathrm{c}$ & $112.50 \mathrm{c}$ & $86.63 \mathrm{a}$ & $55.12 \mathrm{c}$ \\
\hline HD13 103 & $67.14 \mathrm{c}$ & $5.43 \mathrm{~b}$ & $128.92 \mathrm{a}$ & $90.66 \mathrm{a}$ & $63.65 \mathrm{~b}$ \\
\hline HD13 105 & $70.27 \mathrm{~b}$ & $4.39 \mathrm{~d}$ & $104.64 \mathrm{c}$ & $79.71 \mathrm{~b}$ & $50.79 \mathrm{~d}$ \\
\hline HD13 107 & $59.09 \mathrm{c}$ & $5.29 \mathrm{~b}$ & $102.12 \mathrm{c}$ & $75.62 b$ & $44.16 \mathrm{e}$ \\
\hline HD13 115 & $65.10 \mathrm{c}$ & $4.37 \mathrm{~d}$ & $110.34 \mathrm{c}$ & $82.24 b$ & $50.59 \mathrm{~d}$ \\
\hline HD13 123 & $71.04 \mathrm{~b}$ & $3.47 \mathrm{e}$ & $119.32 \mathrm{~b}$ & $89.20 \mathrm{a}$ & $58.99 \mathrm{c}$ \\
\hline HD13 124 & $66.00 \mathrm{c}$ & $5.08 \mathrm{c}$ & $99.92 \mathrm{c}$ & 80.94 b & $53.08 \mathrm{c}$ \\
\hline HD13 125 & $71.31 \mathrm{~b}$ & $4.12 \mathrm{e}$ & $104.22 \mathrm{c}$ & $79.76 \mathrm{~b}$ & $69.76 \mathrm{a}$ \\
\hline HD13 133 & $82.07 \mathrm{a}$ & $6.64 \mathrm{a}$ & $105.62 \mathrm{c}$ & $77.11 \mathrm{~b}$ & $49.92 \mathrm{~d}$ \\
\hline HD13 134 & $62.20 \mathrm{c}$ & $4.55 \mathrm{~d}$ & $106.73 \mathrm{c}$ & $77.96 \mathrm{~b}$ & $46.74 \mathrm{~d}$ \\
\hline HD13 135 & $82.20 \mathrm{a}$ & $4.67 \mathrm{~d}$ & $102.00 \mathrm{c}$ & $76.93 \mathrm{~b}$ & $47.59 \mathrm{~d}$ \\
\hline HD13 136 & $56.82 \mathrm{~d}$ & $4.14 \mathrm{e}$ & $104.01 \mathrm{c}$ & $79.89 \mathrm{~b}$ & $45.63 \mathrm{~d}$ \\
\hline HD13 137 & $69.25 \mathrm{~b}$ & $5.93 \mathrm{~b}$ & $109.82 \mathrm{c}$ & $83.76 \mathrm{a}$ & $49.00 \mathrm{~d}$ \\
\hline HD13 139 & $78.25 \mathrm{~b}$ & $3.69 \mathrm{e}$ & $109.06 \mathrm{c}$ & $86.14 \mathrm{a}$ & $53.17 \mathrm{c}$ \\
\hline HD13 140 & $61.47 \mathrm{c}$ & $3.81 \mathrm{e}$ & $104.00 \mathrm{c}$ & $73.30 \mathrm{c}$ & $52.20 \mathrm{c}$ \\
\hline HD13 141 & $76.74 \mathrm{~b}$ & $5.42 \mathrm{~b}$ & $102.72 \mathrm{c}$ & $79.80 \mathrm{~b}$ & $47.98 \mathrm{~d}$ \\
\hline HD13 145 & $87.81 \mathrm{a}$ & $3.06 \mathrm{f}$ & $113.69 \mathrm{c}$ & $88.59 \mathrm{a}$ & $58.61 \mathrm{c}$ \\
\hline HD13 146 & $76.00 \mathrm{~b}$ & $5.04 \mathrm{c}$ & $103.03 \mathrm{c}$ & $81.75 b$ & $48.70 \mathrm{~d}$ \\
\hline HD13 147 & $67.33 \mathrm{c}$ & $3.72 \mathrm{e}$ & $92.89 \mathrm{~d}$ & $73.51 \mathrm{c}$ & $39.93 \mathrm{e}$ \\
\hline
\end{tabular}

Means followed by the same letters do not differ statistically by the Scott-Knott test at $5 \%$ probability.

The highest values for characters FD, PL and SL were observed for genitors $P$. sublanceolata (88.77-92.23, 40.34-42.85 and 40.83-42.77, respectively), while the lowest dimensions were found for P. foetida var. foetida genotypes (31.03-31.72, 13.97-14.80 and 13.7114.60 , respectively). However, hybrids showed intermediate mean values in relation to parents (59.92-72.06, 26.81-32.85 and 25.22-30.44, respectively) (Table 4).

With regard to CD and SF2, the highest mean values (32.39-43.29 and 12.20-17.21, 
respectively) were found for the hybrids. As to SF1 (10.38-15.44), the hybrids had means higher than those of the P. foetida var. foetida genitors or close to those of one of the parents. The lowest values for characters CD, SF2 and SF1 were determined for the P. sublanceolata genitors (9.19-10.57, 8.28-10.76 and 4.78-7.01, respectively) (Table 4).

For the variables FPL, PW and SW, the highest values were observed for the $P$. sublanceolata genitors (67.10-77.87, 10.56-11.53 and 11.17-12.03, respectively) and the lowest for the P. foetida var. foetida genotypes (18.52-24.83, 5.97-6.30 and 6.06-6.35, respectively). The hybrids showed intermediate values in comparison to the parents or close to one of the genitors (Table 4).

Table 4. Mean values of flower (FD) and corona (CD) diameters, size of first (SF1) and second (SF2) outer series of corona filaments, flower peduncle length (FPL), length (PL) and width (PW) of the petal, and length (SL) and width (SW) of sepal for genitors Passiflora sublanceolata and P. foetida var. foetida and HD13 $\mathrm{F}_{1}$ hybrids.

\begin{tabular}{|c|c|c|c|c|c|c|c|c|c|}
\hline \multirow[t]{2}{*}{ Genotype } & \multicolumn{9}{|c|}{ Characteristics (mm) } \\
\hline & FD & $\mathrm{CD}$ & SF1 & SF2 & FPL & PL & PW & SL & SW \\
\hline P. sublanceolata 1 & $91.88 \mathrm{a}$ & 9.191 & 4.781 & $8.28 \mathrm{i}$ & $77.87 \mathrm{a}$ & $42.85 \mathrm{a}$ & $11.53 \mathrm{a}$ & $42.77 \mathrm{a}$ & $11.76 \mathrm{a}$ \\
\hline P. sublanceolata 2 & $88.77 b$ & 9.781 & $5.44 \mathrm{k}$ & $9.15 \mathrm{~h}$ & $75.85 \mathrm{a}$ & $40.93 \mathrm{~b}$ & $10.62 \mathrm{~b}$ & $40.83 \mathrm{~b}$ & $11.17 \mathrm{~b}$ \\
\hline P. sublanceolata 3 & $89.28 \mathrm{~b}$ & 9.441 & $6.22 \mathrm{j}$ & $10.05 \mathrm{~g}$ & $67.10 \mathrm{~b}$ & $40.34 \mathrm{~b}$ & $11.10 \mathrm{a}$ & $41.52 \mathrm{~b}$ & $11.87 \mathrm{a}$ \\
\hline P. sublanceolata 4 & $92.23 \mathrm{a}$ & $10.57 \mathrm{k}$ & $7.01 \mathrm{i}$ & $10.76 \mathrm{f}$ & $75.59 \mathrm{a}$ & $41.52 \mathrm{~b}$ & $10.56 \mathrm{~b}$ & $42.33 \mathrm{a}$ & $11.54 \mathrm{a}$ \\
\hline P. sublanceolata 5 & $92.22 \mathrm{a}$ & 9.611 & $5.77 \mathrm{k}$ & $8.95 \mathrm{~h}$ & $73.58 \mathrm{a}$ & $41.37 \mathrm{~b}$ & $11.14 \mathrm{a}$ & $42.69 \mathrm{a}$ & $12.03 \mathrm{a}$ \\
\hline P. foetida 6 & $31.72 \mathrm{~h}$ & $28.35 \mathrm{j}$ & $10.83 \mathrm{~g}$ & $10.75 \mathrm{f}$ & $20.26 \mathrm{f}$ & $13.97 \mathrm{f}$ & $5.97 \mathrm{~h}$ & $13.71 \mathrm{~g}$ & $6.06 \mathrm{~h}$ \\
\hline P. foetida 7 & $31.14 \mathrm{~h}$ & $28.03 \mathrm{j}$ & $10.87 \mathrm{~g}$ & $10.56 \mathrm{f}$ & $19.86 \mathrm{f}$ & $14.26 \mathrm{f}$ & $6.30 \mathrm{~h}$ & $14.20 \mathrm{~g}$ & $6.24 \mathrm{~h}$ \\
\hline P. foetida 8 & $31.23 \mathrm{~h}$ & $28.66 \mathrm{j}$ & $10.87 \mathrm{~g}$ & $10.78 \mathrm{f}$ & $18.52 \mathrm{f}$ & $14.79 \mathrm{f}$ & $6.05 \mathrm{~h}$ & $14.60 \mathrm{~g}$ & $6.24 \mathrm{~h}$ \\
\hline P. foetida 9 & $31.03 \mathrm{~h}$ & $28.42 \mathrm{j}$ & $10.55 \mathrm{~h}$ & $10.45 \mathrm{f}$ & $24.81 \mathrm{f}$ & $14.80 \mathrm{f}$ & $6.26 \mathrm{~h}$ & $14.42 \mathrm{~g}$ & $6.35 \mathrm{~h}$ \\
\hline P. foetida 10 & $31.28 \mathrm{~h}$ & $27.81 \mathrm{j}$ & $10.30 \mathrm{~h}$ & $9.84 \mathrm{~g}$ & $24.83 \mathrm{f}$ & $14.41 \mathrm{f}$ & $6.14 \mathrm{~h}$ & $14.29 \mathrm{~g}$ & $6.24 \mathrm{~h}$ \\
\hline HD13 101 & $62.98 \mathrm{f}$ & $34.46 \mathrm{f}$ & $11.73 \mathrm{e}$ & $13.30 \mathrm{e}$ & $60.06 \mathrm{c}$ & $27.04 \mathrm{e}$ & $10.12 \mathrm{~b}$ & $25.22 \mathrm{f}$ & $9.88 \mathrm{~d}$ \\
\hline HD13 102 & $63.27 \mathrm{f}$ & $34.51 \mathrm{f}$ & $11.94 \mathrm{e}$ & $12.48 \mathrm{e}$ & $53.13 \mathrm{~d}$ & $28.43 \mathrm{e}$ & $9.94 \mathrm{~b}$ & $27.09 \mathrm{e}$ & $9.93 \mathrm{~d}$ \\
\hline HD13 103 & $69.46 \mathrm{~d}$ & $36.96 \mathrm{~d}$ & $11.33 \mathrm{f}$ & $13.70 \mathrm{~d}$ & $45.48 \mathrm{~d}$ & $30.47 \mathrm{~d}$ & $8.87 \mathrm{~d}$ & $30.39 \mathrm{c}$ & $8.93 \mathrm{e}$ \\
\hline HD13 105 & $59.92 \mathrm{~g}$ & $35.12 \mathrm{e}$ & $11.90 \mathrm{e}$ & $13.82 \mathrm{~d}$ & $35.59 \mathrm{e}$ & $27.51 \mathrm{e}$ & $9.34 \mathrm{c}$ & $28.25 \mathrm{e}$ & $9.25 \mathrm{e}$ \\
\hline HD13 107 & $62.82 \mathrm{f}$ & $37.69 \mathrm{c}$ & $12.81 \mathrm{~d}$ & $14.35 \mathrm{c}$ & $54.15 \mathrm{~d}$ & $27.92 \mathrm{e}$ & $8.11 \mathrm{e}$ & $27.97 \mathrm{e}$ & $8.78 \mathrm{e}$ \\
\hline HD13 115 & $68.80 \mathrm{~d}$ & $38.91 \mathrm{~b}$ & $12.76 \mathrm{~d}$ & $15.08 \mathrm{~b}$ & $57.83 \mathrm{c}$ & $30.35 \mathrm{~d}$ & $10.40 \mathrm{~b}$ & $29.20 \mathrm{~d}$ & $10.14 \mathrm{~d}$ \\
\hline HD13 123 & $71.74 \mathrm{c}$ & $43.29 \mathrm{a}$ & $14.25 \mathrm{~b}$ & $16.86 \mathrm{a}$ & $52.88 \mathrm{~d}$ & $30.89 \mathrm{~d}$ & $8.99 \mathrm{~d}$ & $29.32 \mathrm{~d}$ & $9.15 \mathrm{e}$ \\
\hline HD13 124 & $60.91 \mathrm{~g}$ & $32.65 \mathrm{~h}$ & $11.32 \mathrm{f}$ & $12.84 \mathrm{e}$ & $36.56 \mathrm{e}$ & $26.81 \mathrm{e}$ & $7.17 \mathrm{f}$ & $28.27 \mathrm{e}$ & $8.31 \mathrm{f}$ \\
\hline HD13 125 & $62.29 \mathrm{f}$ & $34.68 \mathrm{f}$ & $11.12 \mathrm{~g}$ & $13.02 \mathrm{e}$ & $56.57 \mathrm{c}$ & $27.95 \mathrm{e}$ & $8.53 \mathrm{~d}$ & $26.34 \mathrm{f}$ & $8.22 \mathrm{f}$ \\
\hline HD13 133 & $64.90 \mathrm{e}$ & $34.57 \mathrm{f}$ & $12.08 \mathrm{e}$ & $13.01 \mathrm{e}$ & $51.01 \mathrm{~d}$ & $27.63 \mathrm{e}$ & $10.58 \mathrm{~b}$ & $27.52 \mathrm{e}$ & $10.51 \mathrm{c}$ \\
\hline HD13 134 & $72.06 \mathrm{c}$ & $34.26 \mathrm{f}$ & $11.86 \mathrm{e}$ & $13.23 \mathrm{e}$ & $57.03 \mathrm{c}$ & $30.30 \mathrm{~d}$ & $10.60 \mathrm{~b}$ & $28.48 \mathrm{e}$ & $10.64 \mathrm{c}$ \\
\hline HD13 135 & $69.82 \mathrm{~d}$ & $38.87 \mathrm{~b}$ & $13.57 \mathrm{c}$ & $14.74 \mathrm{~b}$ & $64.15 \mathrm{c}$ & $30.18 \mathrm{~d}$ & $8.67 \mathrm{~d}$ & $30.17 \mathrm{c}$ & $9.01 \mathrm{e}$ \\
\hline HD13 136 & $69.29 \mathrm{~d}$ & $43.05 \mathrm{a}$ & $15.44 \mathrm{a}$ & $17.21 \mathrm{a}$ & $63.47 \mathrm{c}$ & $30.73 \mathrm{~d}$ & $8.79 \mathrm{~d}$ & $30.36 \mathrm{c}$ & $8.84 \mathrm{e}$ \\
\hline HD13 137 & $69.50 \mathrm{~d}$ & $35.72 \mathrm{e}$ & $11.38 \mathrm{f}$ & $13.12 \mathrm{e}$ & $61.65 \mathrm{c}$ & $29.38 \mathrm{~d}$ & $10.36 \mathrm{~b}$ & $29.29 \mathrm{~d}$ & $10.12 \mathrm{~d}$ \\
\hline HD13 139 & $64.30 \mathrm{e}$ & $32.59 \mathrm{~h}$ & $10.43 \mathrm{~h}$ & $12.20 \mathrm{e}$ & $54.20 \mathrm{~d}$ & $27.63 \mathrm{e}$ & $8.22 \mathrm{e}$ & $28.53 \mathrm{e}$ & $8.78 \mathrm{e}$ \\
\hline HD13 140 & $68.78 \mathrm{~d}$ & $36.80 \mathrm{~d}$ & $11.34 \mathrm{f}$ & $12.94 \mathrm{e}$ & $60.94 \mathrm{c}$ & $32.85 \mathrm{c}$ & $9.04 \mathrm{~d}$ & $30.44 \mathrm{c}$ & $9.43 \mathrm{e}$ \\
\hline HD13 141 & $65.11 \mathrm{e}$ & $37.43 \mathrm{c}$ & $12.05 \mathrm{e}$ & $14.46 \mathrm{c}$ & $75.96 \mathrm{a}$ & $28.63 \mathrm{e}$ & $9.05 \mathrm{~d}$ & $28.46 \mathrm{e}$ & $9.85 \mathrm{~d}$ \\
\hline HD13 145 & $65.29 \mathrm{e}$ & $36.66 \mathrm{~d}$ & $12.28 \mathrm{e}$ & $13.91 \mathrm{~d}$ & $66.08 \mathrm{~b}$ & $29.93 \mathrm{~d}$ & $9.61 \mathrm{c}$ & $29.51 \mathrm{~d}$ & $9.60 \mathrm{~d}$ \\
\hline HD13 146 & $70.09 \mathrm{~d}$ & $33.58 \mathrm{~g}$ & $10.38 \mathrm{~h}$ & $12.71 \mathrm{e}$ & $50.15 \mathrm{~d}$ & $30.00 \mathrm{~d}$ & $9.20 \mathrm{c}$ & $29.08 \mathrm{~d}$ & $9.05 \mathrm{e}$ \\
\hline HD13 147 & $62.04 \mathrm{f}$ & $32.39 \mathrm{~h}$ & $10.58 \mathrm{~h}$ & $12.88 \mathrm{e}$ & $34.60 \mathrm{e}$ & $27.21 \mathrm{e}$ & $6.83 \mathrm{~g}$ & $27.81 \mathrm{e}$ & $7.47 \mathrm{~g}$ \\
\hline
\end{tabular}

Means followed by the same letters do not differ statistically by the Scott-Knott test at $5 \%$ probability.

\section{Genetic diversity}

The clustering carried out based on the genetic distances of 14 morphologic descriptors resulted in the formation of three groups (Figure 1). The first group was constituted by the five $P$. sublanceolata genitors $(2,3,1,4$, and 5) and the second by the five $P$. foetida var. foetida genitors $(7,8,9,6$, and 10$)$, and the third group was formed by the $20 \mathrm{~F}_{1}$ hybrid genotypes. The characters that contributed most to the genetic divergence according to the Singh (1981) method were FD with $26.27 \%$ and FPL with $26.34 \%$. 


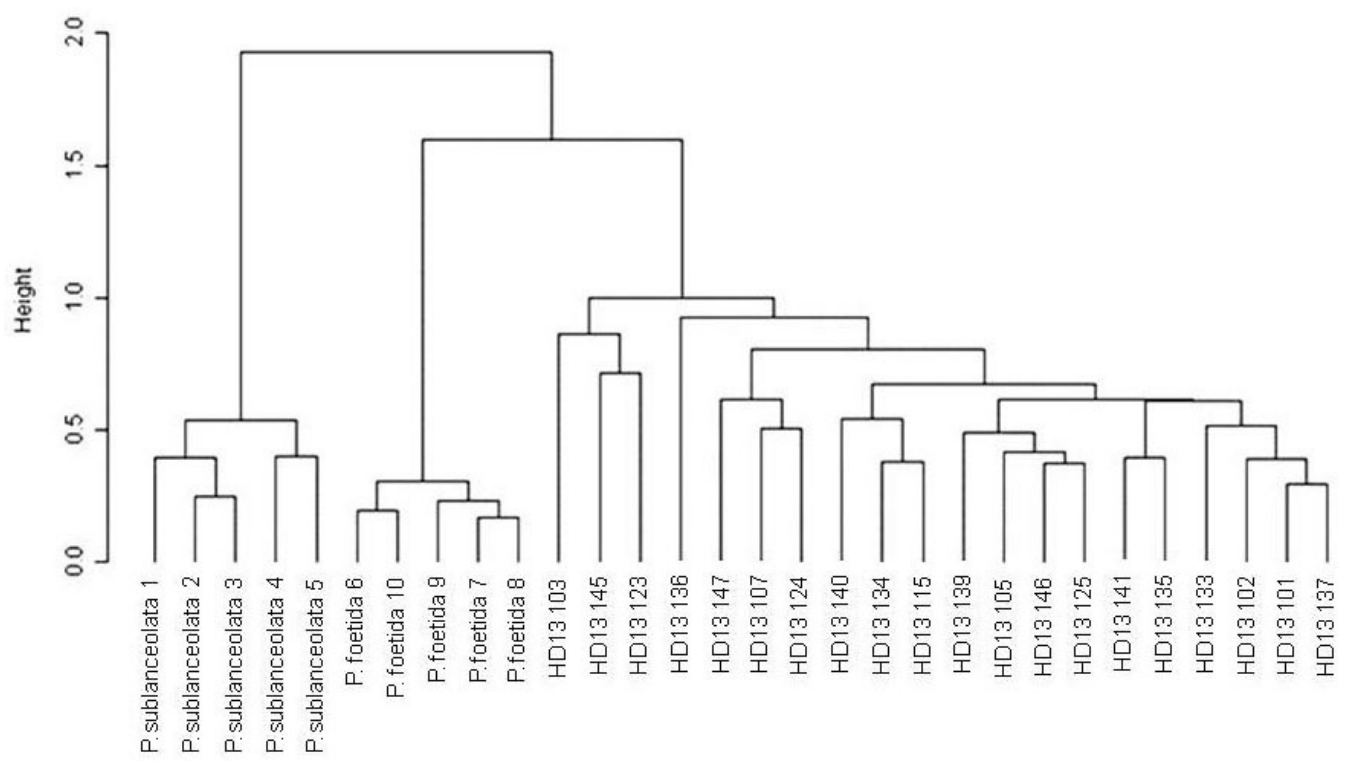

Figure 1. Similarity dendrogram between genitors and hybrids of Passiflora represented by five genotypes of female genitor $P$. sublanceolata, five genotypes of male genitor $P$. foetida var. foetida and twenty $\mathrm{F}_{1}$ hybrid genotypes called HD13, generated by the UPGMA grouping method.

The principal component analysis reduced the 14 descriptors to two principal components that explained the $84 \%$ of the total variance. Component 1 explained $48.9 \%$ of the total variance and component 2 explained $35.12 \%$ (Figure 2). The groups formed by means of principal component analysis did not differ from the UPGMA hierarchical grouping method, displaying the same constitution.

\section{Estimates of genetic parameters}

The $V C_{e}$ estimates showed low values for almost all characteristics evaluated, except for LA (Tables 5 and 6), indicating a good experimental precision. Values obtained for $V C_{o}$ varied from 6.16 to $32.65 \%$ (Tables 5 and 6). All characteristics related to flowers showed high $V C_{g}$ (Table 6), in contrast to the vegetative characteristics, which exhibited very low values, with the exception of SD (Table 5).

The VI observed in this study varied from 0.90 to $15.40 \%$ (Tables 5 and 6 ). The highest values were observed for floral characters (Table 6). In contrast, vegetative characteristics showed VI values less than unity, with the exception of SD and LL (Table 5).

The genotypic determination coefficient $H^{2}$ estimates were higher than $76 \%$ for all characteristics evaluated (Tables 5 and 6 ). The highest estimates were shown by the floral characters, all of them with $H^{2}$ higher than 97\%; the highest values were found for CD (99.89\%) and FD (99.84\%) (Table 6). The lowest estimates were observed for vegetative descriptors, where IL, SD, LL, LW, and LA showed values of 77.30, 95.76, 83.10, and $77.09 \mathrm{~mm}$ and 76.62 $\mathrm{cm}^{2}$, respectively (Table 5). 


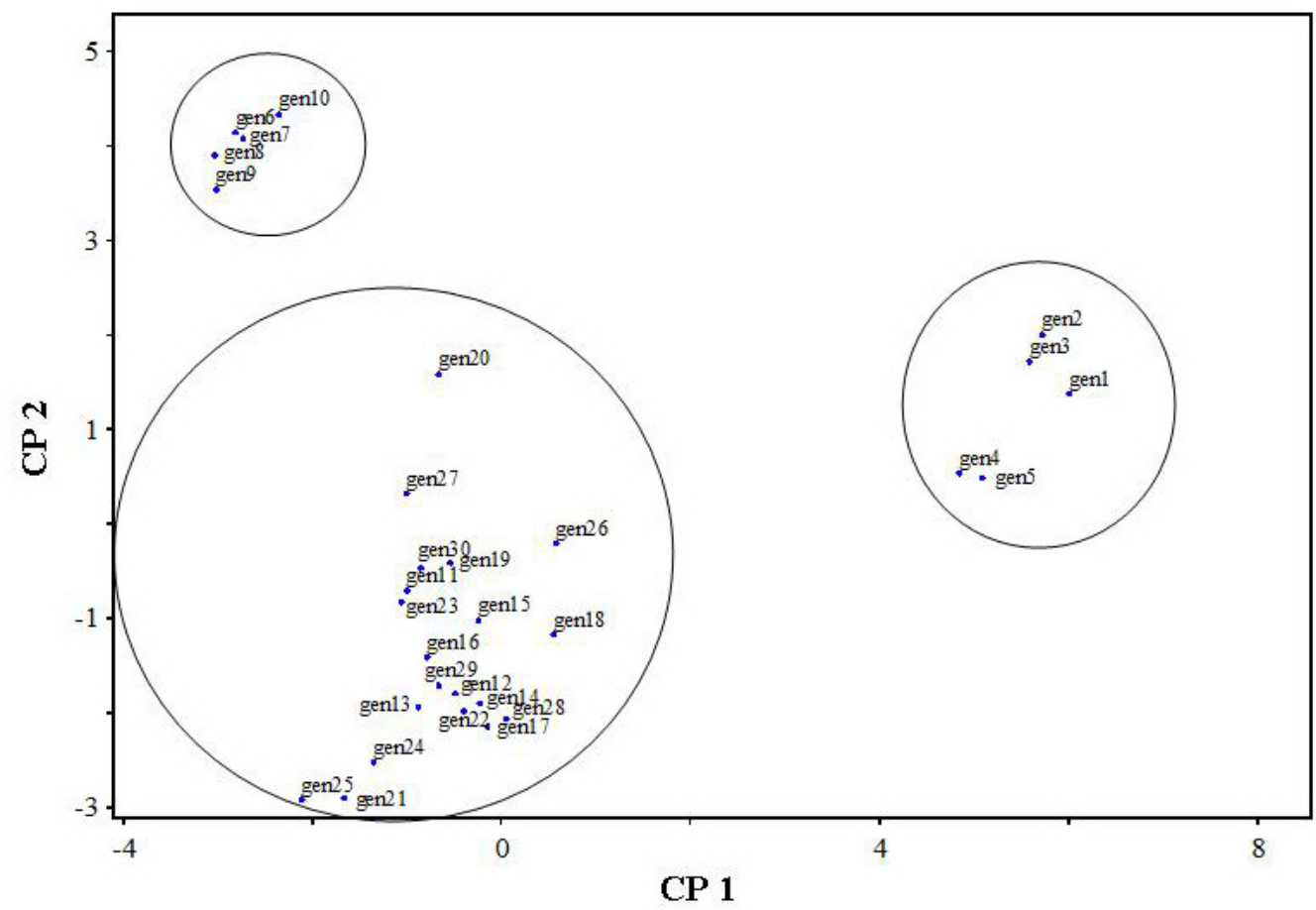

Figure 2. Dispersion of 30 Passiflora genotypes based on two principal components, estimated from 14 quantitative morphological descriptors. gen1-5 $=$ P. sublanceolata; gen6-10 $=$ P. foetida; gen $11=$ HD13 105; gen12 = HD13 101; gen13 = HD13 102; gen14 = HD13 141; gen15 = HD13 146; gen16 = HD13 136; gen17 = HD13 133; gen18 = HD13 134; gen 19 = HD13 107; gen20 = HD13 147; gen21 = HD13 103; gen22 = HD13 115; gen23 = HD13 139; gen24 = HD13 145; gen25 = HD13 123; gen26 = HD13140; gen27 = HD13 124; gen28 = HD13 137; gen29 $=\mathrm{HD} 13$ 135; gen30 $=\mathrm{HD} 13125$.

Table 5. Phenotypic variance estimate $\left(\sigma_{f}^{2}\right)$, genotypic variability $\left(\phi_{g}\right)$, residual variance $\left(\sigma_{e}^{2}\right)$, genotypic determination coefficient $\left(H^{2}\right)$, experimental variation coefficient $\left(V C_{e}\right)$, genotypic variation coefficient $\left(V C_{\mathrm{o}}\right)$, and variation index $(V I)$ for five vegetative characteristics in genitors Passiflora sublanceolata and $P$. foetida var. foetida, and $\mathrm{HD} 13 \mathrm{~F}_{1}$ hybrids.

\begin{tabular}{llcccc}
\hline & \multicolumn{5}{c}{ Characteristics } \\
\cline { 2 - 5 } & $\mathrm{IL}(\mathrm{mm})$ & $\mathrm{SD}(\mathrm{mm})$ & $\mathrm{LL}(\mathrm{mm})$ & LW $(\mathrm{mm})$ & LA $\left(\mathrm{cm}^{2}\right)$ \\
\hline$\sigma_{f}^{2}$ & 35.5442 & 0.6344 & 54.9658 & 28.2217 & 26.4284 \\
$\phi_{g}$ & 27.4781 & 0.6076 & 45.6810 & 21.7569 & 20.2507 \\
$\sigma_{e}^{2}$ & 32.2643 & 0.1075 & 37.1391 & 25.8593 & 24.7107 \\
$H^{2}$ & 77.30 & 95.76 & 83.10 & 77.09 & 76.62 \\
$V C_{e}$ & 9.17 & 9.13 & 6.42 & 6.72 & 11.78 \\
$V C_{g}$ & 8.4684 & 21.7127 & 7.1280 & 6.1698 & 10.6687 \\
$V I$ & 0.92 & 2.37 & 1.11 & 0.91 & 0.90 \\
\hline
\end{tabular}

$\mathrm{IL}=$ internode length; $\mathrm{SD}=$ stem diameter; $\mathrm{LL}=$ leaf length; $\mathrm{LW}=$ leaf width; $\mathrm{LA}=$ leaf area. 
Table 6. Phenotypic variance estimate $\left(\sigma_{f}^{2}\right)$, genotypic variability $\left(\phi_{g}\right)$, residual variance $\left(\sigma_{e}^{2}\right)$, genotypic determination coefficient $\left(H^{2}\right)$, experimental variation coefficient $\left(V C_{e}\right)$, genotypic variation coefficient $\left(V C_{g}\right)$, and variation index $(V I)$ for nine flower characteristics in genitors Passiflora sublanceolata and $P$. foetida var. foetida, and $\mathrm{HD} 13 \mathrm{~F}_{1}$ hybrids.

\begin{tabular}{lccccccccc}
\hline & \multicolumn{7}{c}{ Characteristics (mm) } \\
\cline { 2 - 9 } & FD & CD & SF1 & SF2 & FPL & PL & PW & SL & SW \\
\hline$\sigma_{f}^{2}$ & 312.0045 & 65.1929 & 4.0028 & 2.2123 & 263.34 & 63.2761 & 2.2581 & 66.9895 & 2.6918 \\
$\phi_{g}$ & 311.5107 & 65.1236 & 3.9710 & 2.1658 & 257.176 & 63.1395 & 2.2267 & 66.7922 & 2.6643 \\
$\sigma_{e}^{2}$ & 1.9753 & 0.2769 & 0.1272 & 0.1859 & 24.6864 & 0.5464 & 0.1258 & 0.7892 & 0.1100 \\
$H^{2}$ & 99.84 & 99.89 & 99.20 & 97.89 & 97.65 & 99.78 & 98.60 & 99.70 & 98.97 \\
$V C_{e}$ & 2.23 & 2.12 & 3.74 & 3.83 & 9.92 & 2.61 & 4.05 & 3.14 & 3.66 \\
$V C_{g}$ & 28.1178 & 32.6584 & 20.9317 & 13.1041 & 32.0285 & 28.0679 & 17.0537 & 28.8990 & 18.01 \\
$V I$ & 12.60 & 15.40 & 5.59 & 3.42 & 3.22 & 10.75 & 4.20 & 9.20 & 4.92 \\
\hline
\end{tabular}

$\mathrm{FD}=$ flower diameter; $\mathrm{CD}=$ corona diameter; $\mathrm{SF} 1$ = size of first outer series of corona filaments; SF2 = size of second outer series of corona filaments; FPL = flower peduncle length; PL = petal length; PW = petal width; $\mathrm{SL}=$ sepal length; SW = sepal width.

\section{DISCUSSION}

\section{Genetic diversity based on morphologic characters}

Morphological characterization is an essential step in breeding programs because it allows the monitoring of genetic quality, thus selecting the best genotypes to be used in breeding programs (Radmann and Oliveira, 2003). Based on the means of the morphological characters obtained from the 30 genotypes evaluated, it was possible to detect larger variability for interspecific hybrids. This will provide the breeder a larger variability to be explored in the course of a genetic improvement program for obtaining Passiflora ornamental plants.

The genetic divergence estimates showed a high potential for genotype differentiation, denoting a large genetic variability among the genotypes evaluated. The genetic distance analysis carried out by means of the morphological characters allowed the formation of two groups with greater similarity, formed by the genitors $P$. sublanceolata and $P$. foetida var. foetida, and a third group of segregating individuals, formed by hybrid genotypes.

In group I, represented by the five genotypes of $P$. sublanceolata, plants with higher FD were observed, varying from 88.77 to $92.22 \mathrm{~mm}$, and lower CD, IL and LA dimensions, which varied from 9.19 to $10.57 \mathrm{~mm}, 52.02$ to $61.50 \mathrm{~mm}$ and 31.11 to $39.87 \mathrm{~cm}^{2}$, respectively. This group is formed by pink flowers and delicate foliage (Killip, 1938; Vanderplank, 2000; Ulmer and MacDougal, 2004). The second group, constituted by the five genotypes of $P$. foetida var. foetida, is formed by white flowers and profuse blooming (Killip, 1938; Vanderplank, 2000; Ulmer and MacDougal, 2004). This group is characterized by flowers with lower FD (31.03 to $31.72 \mathrm{~mm}$ ), CD varying from 27.81 to $28.66 \mathrm{~mm}$, IL higher than in the first group, from 57.14 to $62.20 \mathrm{~mm}$, and LA varying from 40.42 to $45.07 \mathrm{~cm}^{2}$. The third group, formed by segregating genotypes, showed a large variability of colors, shapes and sizes, especially with regard to floral characteristics. The colors of the flowers varied from white to light pink (Santos, 2008), and FD showed values intermediate with respect to the genitors, varying from 59.92 to $72.06 \mathrm{~mm}$. The highest CD, IL and LA values were found in this group, varying from 32.39 to $43.29 \mathrm{~mm}, 56.82$ 
to $87.81 \mathrm{~mm}$ and 39.93 to $69.76 \mathrm{~cm}^{2}$, respectively.

In this study, the genotypes formed coherent groups based on morphological characters, allowing the study of genetic diversity. The clear separation between the groups indicated that the quantitative characters were efficient for the discrimination of genitors and hybrids. One of the reasons may be the fact that most of the characteristics evaluated are related to floral characteristics, which are highly heritable (Kobayashi et al., 2007). The hierarchical method and principal component analysis grouped the genotypes identically, showing that the hybrids are genetically closer to P. foetida, which indicates that there is greater expression of genes inherited from this genitor. Results similar to these observed in this study were obtained in a study of morphological diversity with P. tripartita var. mollissima, P. tarminiana and $P$. mixta and their hybrids, which resulted in a clear separation between these species and their hybrids (Primot et al., 2005). The morphological characterization of 11 species of the genus Passiflora, including the two botanical forms of $P$. edulis, the purple and the yellow passion fruit plants, allowed a clear separation between species and also between the two botanical forms of P. edulis (Crochemore et al., 2003).

Data obtained with the population under study favored the identification of superior genotypes, mainly with regard to the FD and CD characteristics, which will provide more advantageous gains in view of their selection. Thus, plants with intermediate FD, higher CD, and lower IL may be selected, backcrossed and/or recombined in order to obtain more attractive plants with regard to floral traits in the course of a breeding program. The results obtained with morphological characterization allow us to predict some of the crosses described below. Thus, aiming to increase corona diameter, we suggest crosses between genotypes of the third group, such as: HD13 136 vs HD13 135, HD13 136 vs HD13 123, HD13 136 vs HD13 115, and HD13 136 vs HD13 140. To recover the pink color of the flowers, some hybrids of group III that are light pink could be backcrossed with $P$. sublanceolata 4 of group I that shows larger CD. Thus, the following crosses are suggested: P. sublanceolata 4 vs HD13 136, P. sublanceolata 4 vs HD13 139, $P$. sublanceolata 4 vs HD13 140, and P. sublanceolata 4 vs HD13 145. There are countless success possibilities if the best genotypes for the desired characteristics are recombined.

\section{Estimates of genetic parameters}

The estimates of the variance components are important since they enable us to determine the genetic control of character and selection potential. When the genotypic variability estimates are analyzed in comparison to phenotypic and experimental variance estimates, the existence of genetic variance is confirmed. Genetic variance is the essential condition for the carrying out plant breeding programs, because it enables the selection and attainment of superior genotypes (Allard, 1960).

The experimental variation coefficients, which give the notion of precision to the experiment when found in essays carried out in the field, may be considered low when they are less than $10 \%$, medium when 10 to $20 \%$, high when 20 to $30 \%$, and very high when greater than $30 \%$ (Pimentel-Gomes, 2000). Therefore, the experimental variation coefficients obtained in this study for all floral characteristics and almost all vegetative characteristics were considered to be low, with values less than $10 \%$. The exception is LA, which showed a $V C_{e}$ of 11.78 , indicating more influence of environmental conditions on this trait. It is worth highlighting that $V C_{e}$ related to floral characters showed values even lower than for vegetative 
characters, indicating less influence of the environment on these characteristics. Other studies with yellow passion fruit resulted in $V C_{e}$ values of $47.13 \%$ for the number of fruits (Gonçalves et al., 2007), considered to be very high. $V C_{e}$ values found in this study showed little environmental influence, suggesting higher stability in the expression of these characteristics in the genotypes evaluated.

Knowledge of the genetic variation coefficient is very important for a breeding program, because it indicates the genetic variation amplitude of a character in view of improvement possibility (Valois et al., 1980). The $V C_{g}$ estimates obtained were high, revealing the existence of a large genetic variability between genotypes, which is useful for the improvement of these ornamental passiflora species, in order to obtain superior genotypes with more attractive floral characteristics. All floral characteristics showed high $V C_{g}$, as opposed to vegetative characteristics, which exhibited very low values, with exception of SD, showing a greater effect of the environment on these characteristics. Similar $V C_{g}$ results for floral characteristics were found for yellow passion fruit with regard to number of fruits, a very satisfactory result for the improvement of this crop, currently going through a period of full expansion in South America (Viana et al., 2004).

The relationship between $V C_{g} / V C_{e}$, called VI, is an important indicator of the success possibilities in obtaining genetic gains by means of selection, showing a favorable situation when values are higher than 1.0 (Vencovsky, 1987). With the exception of the characteristics IL, LW and LA, which attained VI values less than unity, all other characters showed satisfactory variability levels, useful in breeding.

The high experimental precision observed for most of the characters contributes to the attainment of high genotypic determination coefficients, equivalent to broad-sense heritability (Vencovsky and Barriga, 1992). $H^{2}$ values higher than $97 \%$ indicate that the individuals selected for the characteristics FD, CD, SF1, SF2, FPL, PL, PW, SL, and SW showed variability due to the genotype, i.e., more than $97 \%$ of the genetic variability is predominantly due to the genotype. The selection of the superior genotypes based on these characteristics is advisable, where they are the most favorable in breeding programs for obtaining ornamental plants, assuming that the flower is the most attractive product in this market. The highest $H^{2}$ estimate was found for CD (99.89), which is rather satisfactory, since the corona is considered to be one of the most exuberant attributes of passiflora flowers because larger and more colorful corona means a more attractive flower. Twelve floral characteristics were evaluated for radish (Raphanus sativus). Broad-sense heritability values varied from 0.93 to 0.99 , suggesting that the floral morphology is highly heritable (Kobayashi et al., 2007). This result corroborates $H^{2}$ estimates found in this study. Vegetative characteristics IL, LL, LW, and LA showed a lower genotypic determination coefficient, even above $76 \%$, and are more influenced by environmental factors and less indicated for selection.

Heritability results were quite satisfactory because knowing that characteristics related to flowers exhibit higher $H^{2}$ may direct selection based on floral traits, thus favoring the selection of superior, more attractive genotypes. Even though $H^{2}$ is not a direct heritability coefficient $\left(h^{2}\right)$, these high estimates reflect high genetic gain expectations and enable higher efficiency in the selection process.

In general, it may be inferred that, under the conditions of this study, floral characteristics are the most indicated for the selection of more attractive plants for ornamental purposes, because they show more variability, a variation index of more than one, and high genetic de- 
termination coefficients, and because their expression is less influenced by the environment. Thus, simple improvement methods such as mass selection and its derivations may be applied to these characteristics so that satisfactory gains are obtained in subsequent generations. However, the vegetative characteristics IL, LW and LA, which showed a variation less than unity and lower genotypic determination coefficients, need more elaborate selection methods.

These estimates highlight the importance of knowing the character inheritance, mainly when starting a breeding program. This will allow strategies to be designed to obtain genetic gains and maintain an adequate genetic base (Cruz and Carneiro, 2003). Besides, it is important to emphasize that genetic parameter estimates must not be extrapolated to other populations or environmental conditions, because these are specific characteristics for the population under study.

\section{ACKNOWLEDGMENTS}

Research supported by Fundação de Amparo à Pesquisa do Estado da Bahia (FAPESB), Conselho Nacional de Desenvolvimento Científico e Tecnológico (CNPq) and Universidade Estadual de Santa Cruz (UESC). The authors thank Programa de Pós-Graduação em Produção Vegetal and Coordenação de Aperfeiçoamento de Pessoal de Nível Superior (CAPES) for awarding a scholarship to E.A. Santos. Dr. Luiz Carlos Bernacci (Instituto Agronômico, Campinas, SP, Brazil) provided taxonomic identification of the genitors.

\section{REFERENCES}

Abreu PP, Souza MM, Santos EA, Pires MV, et al. (2009). Passion flower hybrids and their use in the ornamental plant market: perspectives for sustainable development with emphasis on Brazil. Euphytica 166: 307-315.

Allard RW (1960). Princípios do Melhoramento Genético das Plantas. Editora Edgard Blücher Ltda, Rio de Janeiro.

Bruckner CH (1997). Perspectivas do Melhoramento Genético do Maracujazeiro. In: Maracujá: Temas Selecionados (Manica I, Bruckner CH and Hoffmann M, eds.). Cinco Continentes Editora, Porto Alegre, 25-46.

Cerqueira-Silva CB, Cardoso-Silva CB, Conceicao LD, Nonato JV, et al. (2009). Comparison of coefficients and distance measurements in passion fruit plants based on molecular markers and physicochemical descriptors. Genet. Mol. Res. 8: 870-879.

Cervi AC (2005). Espécies de Passiflora L. (Passifloraceae) publicadas e descritas nos últimos 55 anos (1950-2005) na América do Sul e principais publicações brasileiras. Estudos Biol. 27: 19-24.

Cervi AC (2006). O gênero Passiflora L. (Passifloraceae) no Brasil, espécies descritas após o ano de 1950. Fontqueria 16: $1-5$.

Crochemore ML, Molinari HBC and Vieira LGE (2003). Genetic diversity in passion fruit (Passiflora spp.) evaluated by RAPD markers. Braz. Arch. Biol. Technol. 46: 521-527.

Cruz CD (2006). Programa Genes - Estatística Experimental e Matrizes. Editora UFV, Viçosa.

Cruz CD and Carneiro PCS (2003). Modelos Biométricos Aplicados ao Melhoramento Genético. Editora UFV, Viçosa.

Faleiro FG, Junqueira NTV and Braga MF (2005). Germoplasma e Melhoramento Genético do Maracujazeiro - Desafios da Pesquisa. In: Maracujá: Germoplasma e Melhoramento Genético (Faleiro FG, Junqueira NTV and Braga MF, eds.). Embrapa Cerrados, Planaltina, 187-210.

Gonçalves GM, Viana AP, Neto FVB and Pereira MG (2007). Seleção e herdabilidade na predição de ganhos genéticos em maracujá-amarelo. Pesq. Agropec. Bras. 42: 193-198.

Killip EP (1938). The American species of Passifloraceae. Field Mus. Nat. Hist. Bot. Ser. 19: 1-613.

Kobayashi K, Horisaki A, Niikura S and Ohsawa R (2007). Diallel analysis of floral morphology in radish (Raphanus sativus L.). Euphytica 158: 153-165.

Manica I (1981). Fruticultura 1: Maracujá. Editora Ceres, São Paulo.

Meletti LMM, Soares-Scott MD, Bernacci LC and Passos IRS (2005). Melhoramento Genético do Maracujá: Passado e Futuro. In: Maracujá: Germoplasma e Melhoramento Genético (Faleiro FG, Junqueira NTV and Braga MF, eds.). 
Embrapa Cerrados, Planaltina, 55-78.

Moraes MC, Geraldi IO, Matta FP and Vieira MLC (2005). Genetic and phenotypic parameter estimates for yield and fruit quality traits from a single wide cross in yellow passion fruit. HortScience 40: 1978-1981.

Nunes TS and Queiroz LP (2007). Uma nova espécie de Passiflora L. (Passifloraceae) para o Brasil. Acta Bot. Bras. 21: 499-502.

Peixoto M (2005). Problemas e Perspectivas do Maracujá Ornamental. In: Maracujá: Germoplasma e Melhoramento Genético (Faleiro FG, Junqueira NTV and Braga MF, eds.). Embrapa Cerrados, Planaltina, 457-464.

Pimentel-Gomes F (2000). Curso de Estatística Experimental, 14a ed. Gráfica Degaspari, Piracicaba.

Primot S, D’Eeckenbrugge GC, Rioux V and Pérez JAO (2005). Variación morfológica de tres especies de curubas (Passiflora tripartita var. mollissima, P. tarminiana y P. mixta) y sus híbridos en el valle del cauca (Colombia). Rev. Bras. Frutic. 22: 467-471.

Radmann EB and Oliveira RP (2003). Caracterização de cultivares apirênicas de citros de mesa por meio de descritores morfológicos. Pesq. Agropecu. Bras. 38: 1123-1129.

Rushing F (2003). Tough Plants for Southern Gardens: Low Care, No Care, Tried and True Winners. Cool Springs Press, Tennessee.

Santos EA (2008). Melhoramento de Passifloras para Ornamentação Utilizando Passiflora palmeri var. sublanceolata, Passiflora foetida var. foetida e Híbridos $\mathrm{F}_{1}$ Ornamentais: Confirmação via RAPD, Parâmetros Genéticos e Efeitos do Sombreamento. Master's thesis, UESC, Ilhéus.

Silva MGM, Viana AP, Goncalves GM and Amaral Junior AT (2009). Seleção recorrente intrapopulacional no maracujazeiro-amarelo: alternativa de capitalização de ganhos genéticos. Cienc. Agr. 33: 170-176.

Singh D (1981). The relative importance of characters affecting genetic divergence. Indian J. Genet. Plant. Breed. 41: 237-245.

Ulmer T and MacDougal JM (2004). Passiflora: Passionflowers of the World. Timber Press, Portland.

Valois ACC, Schmidt GS and Sanotto MD (1980). Análise de Qualidade e Quantidade de Grãos em População de Milho (Zea mays L). Master's thesis, USP, São Paulo.

Vanderplank J (2000). Passion Flowers. 3th edn. The MIT Press, Cambridge.

Vencovsky R (1987). Herança Quantitativa. In: Melhoramento e Produção do Milho (Paterniani E and Viegas GP, eds.). $2^{\mathrm{a}}$ ed. Fundação Cargill, Campinas, 137-214.

Vencovsky R and Barriga P (1992). Genética Biométrica Aplicada ao Fitomelhoramento. Sociedade Brasileira de Genética, Ribeirão Preto.

Viana A, Souza M, Araújo I, Corrêa R, et al. (2010). Genetic diversity in Passiflora species determined by morphological and molecular characteristics. Biol. Plant. 54: 535-538.

Viana AJ (2009). Delimitação entre as Espécies Passiflora edulis Sims e Passiflora sp. Nativa da Bahia com Base em Características Citogenéticas, Moleculares e Morfológicas. Master's thesis, UESC, Ilhéus.

Viana AP, Pereira TNS, Pereira MG and Amaral Júnior AT (2004). Parâmetros genéticos em populações de maracujazeiroamarelo. Rev. Ceres 51: 541-551. 